\title{
Overexpression of Soybean Isoflavone Reductase (GmIFR) Enhances Resistance to Phytophthora sojae in Soybean
}

\author{
Qun Cheng ${ }^{1 \dagger}$, Ninghui $\mathrm{Li}^{1,2 \dagger}$, Lidong Dong ${ }^{1 \dagger}$, Dayong Zhang ${ }^{1}$, Sujie Fan ${ }^{1}$, Liangyu Jiang ${ }^{1}$, \\ Xin Wang ${ }^{1,3}$, Pengfei $X u^{1 *}$ and Shuzhen Zhang ${ }^{1 *}$ \\ ${ }^{1}$ Key Laboratory of Soybean Biology of Chinese Education Ministry, Soybean Research Institute, Northeast Agricultural \\ University, Harbin, China, ${ }^{2}$ Jiamusi Branch Academy of Heilongjiang Academy of Agricultural Sciences, Jiamusi, China, \\ ${ }^{3}$ Heilongjiang Academy of Land Reclamation Sciences, Harbin, China
}

OPEN ACCESS

Edited by:

Sylvain Jeandroz,

Agrosup Dijon, France

Reviewed by:

Raimund Tenhaken,

University of Salzburg, Austria

Haitao Shi,

Hainan University, China

${ }^{*}$ Correspondence: Pengfei $X_{U}$

xupengfei@neau.edu.cn;

Shuzhen Zhang

zhangshuzhen@neau.edu.cn

${ }^{\dagger}$ These authors have contributed equally to this work.

Specialty section: This article was submitted to

Plant Physiology,

a section of the journal

Frontiers in Plant Science

Received: 30 July 2015 Accepted: 05 November 2015 Published: 23 November 2015

Citation:

Cheng Q, Li N, Dong L, Zhang D, Fan S, Jiang L, Wang $X, X \cup P$ and

Zhang S (2015) Overexpression of

Soybean Isoflavone Reductase (GmIFR) Enhances Resistance to

Phytophthora sojae in Soybean.

Front. Plant Sci. 6:1024.

doi: 10.3389/fpls.2015.01024
Isoflavone reductase (IFR) is an enzyme involved in the biosynthetic pathway of isoflavonoid phytoalexin in plants. IFRs are unique to the plant kingdom and are considered to have crucial roles in plant response to various biotic and abiotic environmental stresses. Here, we report the characterization of a novel member of the soybean isoflavone reductase gene family GmIFR. Overexpression of GmIFR transgenic soybean exhibited enhanced resistance to Phytophthora sojae. Following stress treatments, GmIFR was significantly induced by $P$. sojae, ethephon (ET), abscisic acid (placeCityABA), salicylic acid (SA). It is located in the cytoplasm when transiently expressed in soybean protoplasts. The daidzein levels reduced greatly for the seeds of transgenic plants, while the relative content of glyceollins in transgenic plants was significantly higher than that of non-transgenic plants. Furthermore, we found that the relative expression levels of reactive oxygen species (ROS) of transgenic soybean plants were significantly lower than those of non-transgenic plants after incubation with $P$. sojae, suggesting an important role of GmIFR might function as an antioxidant to reduce ROS in soybean. The enzyme activity assay suggested that GmIFR has isoflavone reductase activity.

Keywords: Glycine max, isoflavone reductase, Phytophthora sojae, isoflavonoid, gene expression, antioxidant properties

\section{INTRODUCTION}

A major response of soybean to attack by fungal pathogens and oomycetes is production of isoflavonoid phytoalexin glyceollins (Partridge and Keen, 1977; Yoshikawa et al., 1978; Banks and Dewick, 1983; Ng et al., 2011; Kim et al., 2012). They are valuable secondary metabolities produced primarily in leguminous plants and are rarely found in other plant families (Wang et al., 2006; Kim et al., 2010b), and are synthesized by the isoflavonoid branch of the central phenylopropanoid pathway (Ng et al., 2011). Moreover, glyceollins in general protect plant tissues from environmental challenge possibly by reducing the oxidative damage induced by stress factors; therefore, the compounds can possess considerable cellular antioxidant properties (Nwachukwu et al., 2013). A huge variety of enzymes are thought to be involved in their biosynthetic pathways (Somerville and Somerville, 1999). Isoflavone reductase (IFR) is identified as a crucial enzyme 
involved in the synthesis of the glyceollins from daidzein (Graham et al., 1990; Oliver et al., 2003), and catalyzes a stereospecific NADPH-dependent reduction to (3R)-isoflavanone (Guo et al., 1994; Cooper et al., 2002). In addition, IFR is a monomeric, cytosolic reductase, and the enzyme can use $2^{\prime}$-hydroxydaidzein, $2^{\prime}$-hydroxyformononetin, and $2^{\prime}$-hydroxygenistein as substrates in soybean (Wang et al., 2006).

IFRs are members of a large protein family, and their cDNAs cloned from leguminous plants such as alfalfa (Medicago sativa; Paiva et al., 1991), pea (Pisum sativum; Sun et al., 1991), kidney bean (Phaseolus vulgaris; Rípodas et al., 2013) share high levels of sequence identity, and they participate specifically in isoflavonid phytoalexin biosynthesis. IFR was first identified as a key enzyme involved in the latter part of the isoflavonoid phytoalexins pathway in alfalfa (Paiva et al., 1991). In pea, the enzyme has been purified and used to raise polyclonal antibodies (Sun et al., 1991). The levels of IFR in response to Ascochyta rabiei in chickpea were higher than those of control, suggesting that IFR may play a role in determining resistance to fungal (Daniel et al., 1990). In kidney bean, the reduction of IFR levels affected the growth, lateral root elongation and the number of nodules developed (Rípodas et al., 2013). However, beyond the purification to apparent homogeneity of IFR from elicitor-challenged soybean cell cultures and some physical and kinetic properties of this enzyme (Fischer et al., 1990; Wang et al., 2006), the gene function is still unclear in soybean.

In addition, IFR-like (IRL) proteins from non-leguminous plants, which are also part of the IFR family (Kim et al., 2010b), have also been cloned from tobacco (Nicotiana tabacum; Shoji et al., 2002), rice (Oryza sativa; Kim et al., 2003), Arabidopsis (Babiychuk et al., 1995), Ginkgo (Ginkgo biloba; Cheng et al., 2013), etc. They show amino acid sequence homology with those of legumes IFRs. Moreover, several IRL proteins have been implicated in response to biotic or abiotic stresses (Babiychuk et al., 1995; Lers et al., 1998; Shoji et al., 2002; Kim et al., 2003, 2010b). For instance, OsIRL gene was induced with an expression pattern similar to methyl jasmonic acid (MeJA) induction after Pyricularia grisea inoculation of rice (Kim et al., 2003). It has been proposed that AtIRL might have an important role as an antioxidant in yeast (Babiychuk et al., 1995). A recent study showed that OsIRL may behave as an antioxidant in response to reactive oxygen species (ROS) in suspension-cultured cells as well as during root development in rice (Kim et al., 2010b).

In a previous study, a cDNA library enriched for mRNAs encoding ESTs that increased in abundance during infection with Phytophthora sojae was constructed by suppression subtractive hybridization from leaf tissues of a high resistant soybean cultivar "Suinong 10," and an EST homologous to an isoflavone reductase from white lupin (Lupinus albus) was identified to be up-regulated by microarray and real-time PCR (Xu et al., 2012). In this study, the full-length EST, designated GmIFR (GenBank accession no. NM_001254100, NCBI protein no. NP_001241029), was isolated through RT-PCR from "Suinong 10 " soybean, and the transgenic soybean plants over-expressing GmIFR gene under the control of $35 \mathrm{~S}$ promoter were produced. The expression patterns of GmIFR induced under biotic stresses were also examined. Moreover, the function of GmIFR and the content of daidzein, genistein, glycitein, the relative content of glyceollins and the ROS in transgenic plants were investigated. GmIFR protein could catalyze a distinct NADPH-dependent oxidoreductase reaction by enzyme activity assay, suggesting that GmIFR has isoflavone reductase activity. Together, we report insights into the function of an isoflavone reductase (IFR) in soybean, namely GmIFR, in host responses to $P$. sojae.

\section{MATERIALS AND METHODS}

\section{Plant Materials and Stress Treatments}

The soybean cultivar "Suinong10," resistant to the predominant race 1 of $P$. sojae in Heilongjiang, China (Zhang et al., 2010), was used in this study. The seeds of "Suinong10" were grown with a photoperiod of $16 / 8 \mathrm{~h}$ light/dark and maintained at $22^{\circ} \mathrm{C}$ with $70 \%$ relative humidity in the greenhouse. Fourteen days after planting, seedlings at the first-node stage (V1; Fehr et al., 1971) were used for various treatments.

For abiotic treatments, the seedlings were exposed to one of the five different stresses, namely wounding, ET, ABA, SA, or MeJA. For wounding treatment, the edges of leaves were cut by about $0.2 \mathrm{~cm}$ with scissors and incubated at room temperature for $0,1,3,6,9,12$ or $24 \mathrm{~h}$; the intact leaves of soybean were used as controls. The ABA $(200 \mu \mathrm{M})$, SA $(2 \mathrm{mM})$, and MeJA $(100 \mu \mathrm{M})$ were dissolved in $0.01 \%$ Tween 20 and sprayed onto young leaves for $0,1,3,6,9,12$ or $24 \mathrm{~h}$. Ethylene treatment was performed with a concentration of $200 \mu \mathrm{L} \mathrm{L}^{-1}$ by injection of gaseous ethylene in a sealed plexiglass chamber for $0,1,3,6,9,12$, or $24 \mathrm{~h}$. The control leaves were sprayed with an equivalent volume of $0.01 \%$ (v/v) Tween 20.

For $P$. sojae treatment, the soybean plants were inoculated with P. sojae zoospores following the method described by Ward et al. (1979) and Morris et al. (1991) with minor modifications. Mock inoculations were carried out with equivalent amounts of sterile water. Zoospores were developed with the procedure of Ward et al. (1979), and the concentration was estimated using hemacytometer to approximately $1 \times 10^{5}$ spores $\mathrm{mL}^{-1}$. The unifoliolate leaves were also treated for $0,3,6,9,12,24,36,48,64$, $72 \mathrm{~h}$. "Dongnong50" soybean, which was susceptible to P. sojae race 1, obtained from the Key Laboratory of Soybean Biology in Chinese Ministry of Education, Harbin, was used for gene transformation experiments.

\section{Isolation of the GmIFR Gene}

A suppression subtractive hybridization library coupled with cDNA microarrays was queried using a soybean EST encoding an EST homologous to an isoflavone reductase from white lupin (L. albus), previously shown to be upregulated in "Suinong 10" soybean inoculated with P. sojae (Xu et al., 2012). Here, the full-length cDNA (tremed GmIFR, GenBank accession no. NM_001254100, NCBI protein no. NP_001241029) of the EST was amplified by RT-PCR from cDNA of "Suinong 10" using the primer pairs GmIFRF and GmIFRR (Supplementary Table S1). PCR was performed as follows: $94^{\circ} \mathrm{C}$ for $5 \mathrm{~min}$, followed 30 cycles of $94^{\circ} \mathrm{C}$ for $30 \mathrm{~s}, 60^{\circ} \mathrm{C}$ 
for $30 \mathrm{~s}$, and $72^{\circ} \mathrm{C}$ for $90 \mathrm{~s}$, with a final extension at $72^{\circ} \mathrm{C}$ for $10 \mathrm{~min}$. The amplification product was gel purified and cloned into the pMD18-T vector (TaKaRa, Dalian, China). An analysis of protein structure was performed using Smart (http://smart. embl-heidelberg.de/). Sequence alignments were performed using DNAMAN software (http://www.lynnon.com/). A phylogenetic analysis of GmIFR and various heterologous IFR proteins was performed using MEGA4 software (Tamura et al., 2007).

\section{Quantitative RT-PCR Analysis}

Quantitative real-time PCR analysis was performed to determine the transcript abundance of GmIFR. Total RNA was isolated from "Suinong 10" soybean leaves using Trlzol reagent (Invitrogen, Shanghai, China). The synthesis of cDNA was conducted using an oligo(dT) primer and a M-MLV reverse transcriptase kit (Takara, Dalian, China) according to the manufacturer's instructions. qRT-PCR was performed on a CFX96 Touch $^{\mathrm{TM}}$ Real-Time PCR machine (Biobad, USA) using the real-time PCR kit (ToYoBo, Japan). DNA accumulation was measured using SYBR Green as the reference dye. The soybean housekeeping gene Gmactin4 (GenBank accession no. AF049106) was used as the internal control (see Supplementary Table S1 for primer sequences). For tissue distribution analysis, the transcript level of GmEF1 gene (GenBank accession no. NM_001248778) was used as quantitative control (see Supplementary Table $\mathbf{S} 1$ for primer sequence). The relative expression of target gene in different tissues of soybean was calculated using the $2^{-\Delta \Delta C T}$ method. For each sample, three biological replicates were analyzed with their respective technical replicates.

\section{Subcellular Localization of GmIFR Protein}

To determine the subcellular localization of GmIFR, the coding region of GmIFR was fused to the N-terminus of GFP under the control of the CaMV 35S promoter in the PCAMBIA1302 vector. Soybean protoplasts were obtained according to the method described by Lin (1983). Soybean protoplast transformation described by Yoo et al. (2007) was performed with minor modifications.

\section{Expression and Purification of Fusion Protein}

The full-length cDNA of GmIFR was fused to the N-terminus of the $6 \times$ His-tag, at the NcoI and XhoI restriction sites of the vector pET28a (+) (Novagen, Germany). The recombinant fusion plasmid was expressed into Transetta (DE3) cells (TransGen Biotech, China). His-tagged proteins were induced with $0.5 \mathrm{mM}$ isopropyl- $\beta$-D-thiogalactoside (IPTG) at $37^{\circ} \mathrm{C}$ for $4 \mathrm{~h}$. The fusion protein was purified at room temperature and quantified according to the pET System Manual (Novagen). The fusion GmIFR protein was subsequently analyzed by sodium dodecyl sulfate polyacrylamide gel electrophoresis (SDS-PAGE) and western blotting using anti-His antibody.

\section{Enzyme Assays}

In order to determine whether GmIFR has isoflavone reductase activity, the enzyme assays of GmIFR was analyzed. The fusion protein was used in the enzyme assays. GmIFR activity was analyzed according to the method described by Paiva et al. (1991) with minor modifications. High Performance Liquid Chromatography (HPLC) was used to separate the substrate and product.

\section{Plasmid Construction and Transformation of Soybean}

For gene overexpression analysis, the full length GmIFR coding region was amplified with gene specific primers GmIFRTF and GmIFRTR (Supplementary Table S1). The PCR conditions were as follows: $94^{\circ} \mathrm{C}$ for $2 \mathrm{~min}$ followed by 30 cycles at $94^{\circ} \mathrm{C}$ for $30 \mathrm{~s}, 55^{\circ} \mathrm{C}$ for $30 \mathrm{~s}$, and $72^{\circ} \mathrm{C}$ for $1 \mathrm{~min}$ and a final extension at $72^{\circ} \mathrm{C}$ for $10 \mathrm{~min}$. Then the GmIFR open reading frame was cloned into the vector pCAMBIA3301 under the control of a CaMV35S promoter. The constructs were transferred into the Agrobacterium tumefaciens strain LBA4404 via tri-parental mating. For “Dongnong 50" soybean transformation, the cotyledonary nodes were used as explants for the transformation using the Agrobacterium-mediated transformation method described by Paz et al. (2004). Transgenic soybean plants (T7) were indentified by PCR amplification and southern hybridization using the DIG High Prime DNA Labeling and Detection Starter Kit II (Roche Cat., Germany), and they were developed to T8 transgenic soybean plants for further analysis.

\section{Pathogen Response Assays of Transgenic Soybean Plants}

To investigate whether the GmIFR-transformed plants resist pathogen infection, artificial inoculation procedures were performed according to the methods described by Dou et al. (2003) and Morrison and Thorne (1978) with some modifications. The living cotyledons of three T8 transgenic soybean plants (numbered T8-80, T8-88, and T8-96) were treated with a $P$. sojae inoculum. For infection assays, three biological replicates were analyzed with their respective technical replicates. The living cotyledons were incubated in a mist chamber at $25^{\circ} \mathrm{C}$ with $90 \%$ relative humidity under a $14 \mathrm{~h}$ photoperiod at a light intensity of $350 \mu \mathrm{mol}$ photons $\mathrm{m}^{-1} \mathrm{~s}^{-1}$ for investigation. The cotyledons of non-transformed plants were used as controls. Disease symptoms on each cotyledon were observed and photographed after inoculation using a Nikon D7000 camera.

\section{Measurement of Reactive Oxygen Species (ROS) Generation}

To investigate whether the GmIFR-transformed plants respond to oxidative stresses, the hypocotyls of three T8 transgenic soybean plants (numbered T8-31, T8-39, and T8-47) and nontransgenic soybean plants were treated with $P$. sojae zoospores of approximately $1 \times 10^{5}$ spores $\mathrm{mL}^{-1}$ for $0,3,6,12,24$, $48 \mathrm{~h}$ following the method described by Ward et al. (1979) and Morris et al. (1991) with minor modifications. The ROS were measured according to the instructions supplied with the Reactive Oxygen Species Assay Kit (Beyotime Institute 
of Biotechnology, Haimen, China). In this kit, the nonfluorescent probe $2^{\prime}, 7^{\prime}$-dichlorofluorescein diacetate (H2DCFDA) passively diffuses into cells and is deacetylated to form nonfluorescent $2^{\prime}, 7^{\prime}$-dichlorofluorescein (DCFH). DCFH reacts with ROS to form the fluorescent product DCF, which is trapped inside the cells. Fluorescence was detected at $485 \mathrm{~nm}$ for excitation and $530 \mathrm{~nm}$ for emission with a fluorescence microplate reader (Bio-TEK, USA; Qian et al., 2009).

\section{Isoflavone and Glyceollins Analysis}

Approximately $0.1 \mathrm{~g}$ sample of seeds (numbered S-T8-80, ST8-88, S-T8-96) developed from T8-80, T8-88 and T8-96 transgenic soybean plants, and that of non-transgenic soybean plants were ground to a fine power using a commercial coffee grinder. Daidzein, genistein, and glycitein were extracted from flour and separated using HPLC as described previously (Zeng et al., 2009). Measurements were done as micrograms of isoflavone per gram of seeds plus and minus the standard deviations.

Glyceollins were extracted from the seeds (namely S-T880, S-T8-88, S-T8-96) developed from T8-80, T8-88, and T8-96 transgenic soybean plants and non-transgenic soybean with $80 \%$ ethanol following the method described by Boue et al. (2000) and isolated using HPLC as described previously (Zeng et al., 2009).

\section{RESULTS}

\section{Isolation and Bioinformatic Analysis of GmIFR}

The full-length cDNA sequence of GmIFR (GenBank Accession No. NM_001254100) was isolated from total RNA of "Suinong10" soybean by RT-PCR and cloned into pMD$18 \mathrm{~T}$ vector. Sequence analysis showed that GmIFR has an open reading frame (ORF) of $939 \mathrm{bp}$ and encodes a polypeptide of 312 amino acids (Supplementary Figure S1) with a predicted molecular mass of $34.92 \mathrm{kDa}$ and a isoelectric point (pI) of 6.33. The deduced protein has a central 107 amino acid NAD (P) domain (Supplementary Figure S1). The predicted threedimensional model of the GmIFR consists of $13 \alpha$-helices and $10 \beta$-strands (Supplementary Figure S2A). To further explore the evolutionary relationship among plant NAD (P) proteins, a phylogenetic tree was constructed using MEGA 4.0 (Tamura et al., 2007) based on the amino acid sequences. Alignment and phylogenetic tree analysis of leguminous plants sequences revealed that GmIFR has $44-94 \%$ identity for overall amino acid sequence to Phaseolus vulgaris PvIFR, Cicer arietinum CaIFR, Medicago truncatula MtIRF, Lotus japonicus LjIFR, Glycine soja GsIFR, Medicago sativa MsIFR, Pisum sativum PsIFR (Supplementary Figure S2B). Analysis of the conserved NAD (P) domain of 107 aa showed that GmIFR shared 86-90\% amino acid identity with others (Supplementary Figure S2C). The analysis of homologs of GMIFR in the soybean genome, based on data obtained from the Phytozome database (http:// www.phytozome.net/ soybean), indicated that the two genes were clustered on two linkage groups, namely one each on $\mathrm{Gm}$ 01 and $\mathrm{Gm} 09$ and had three introns.

\section{Accumulation of the GmIFR Transcript under Different Stress Treatments}

To determine the expression pattern of GMIFR, quantitative realtime reverse transcription-PCR (qRT-PCR) was performed to examine the transcript levels of GmIFR in "Suinong 10" soybean plant. The examination of tissue-specific transcript abundance in "Suinong 10" soybean showed that GMIFR was constitutively and highly expressed in the cotyledons, followed by roots, stems, and leaves (Figure 1). Quantitative real-time PCR showed that GmIFR was responsive to ET, SA, MeJA, ABA, wounding and $P$. sojae treatments (Figure 2). GmIFR mRNA rapidly increased under ET and SA treatments, reaching a maximum level at $12 \mathrm{~h}$ after the treatment followed by a rapid decline (Figure 2). Under wounding, ABA, MeJA, and P. sojae treatments, GmIFR mRNA accumulated and reached a maximum level at 1, 3, 9 and $48 \mathrm{~h}$, respectively (Figure 2 ).

\section{Subcellular Localization of the GmIFR Protein}

The Psort program predicted a cytoplosmic localization of GmIFR with $45 \%$ certainty (http://psort.hgc.jp/form.html). To determine the subcellular localization of GmIFR protein, GmIFR-GFP (for green fluorescent protein) fusion protein driven by the cauliflower mosaic virus $35 \mathrm{~S}$ promoter was introduced into soybean protoplast by transient transformation. Analysis of the subcellular localization of the GmIFR-GFP fusion proteins by confocal laser scanning microscopy revealed that a strong fluorescent signal derived from GFP alone was observed in the cytoplasm, nuclei and cell membrane, whereas transformed cells carrying GmIFR-GFP showed a strong green fluorescence signal in the cytoplasm (Figure 3), demonstrating the cytoplasm localization of GMIFR.

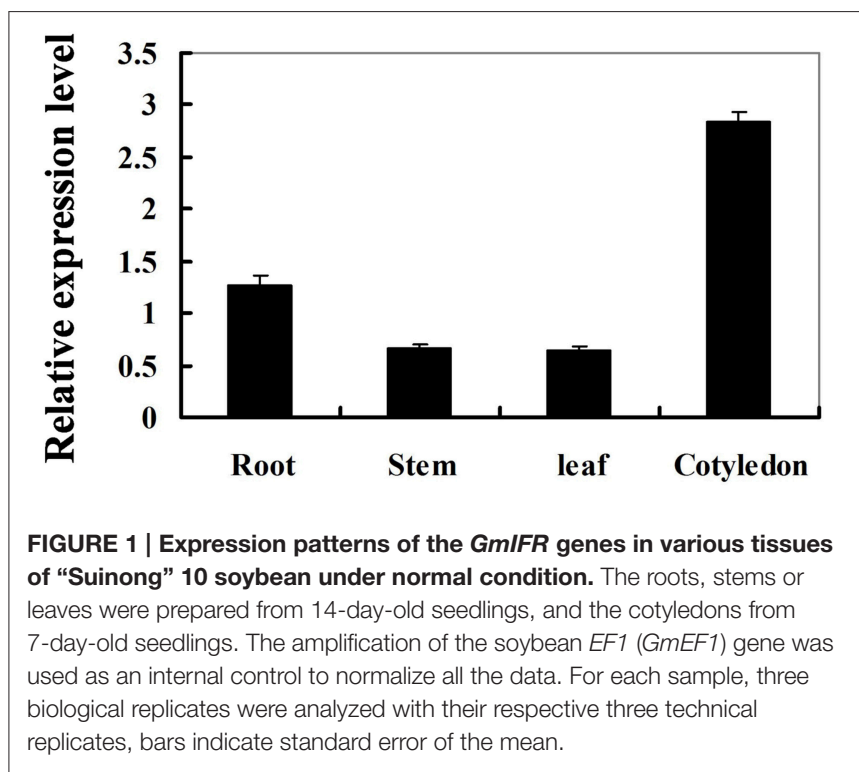



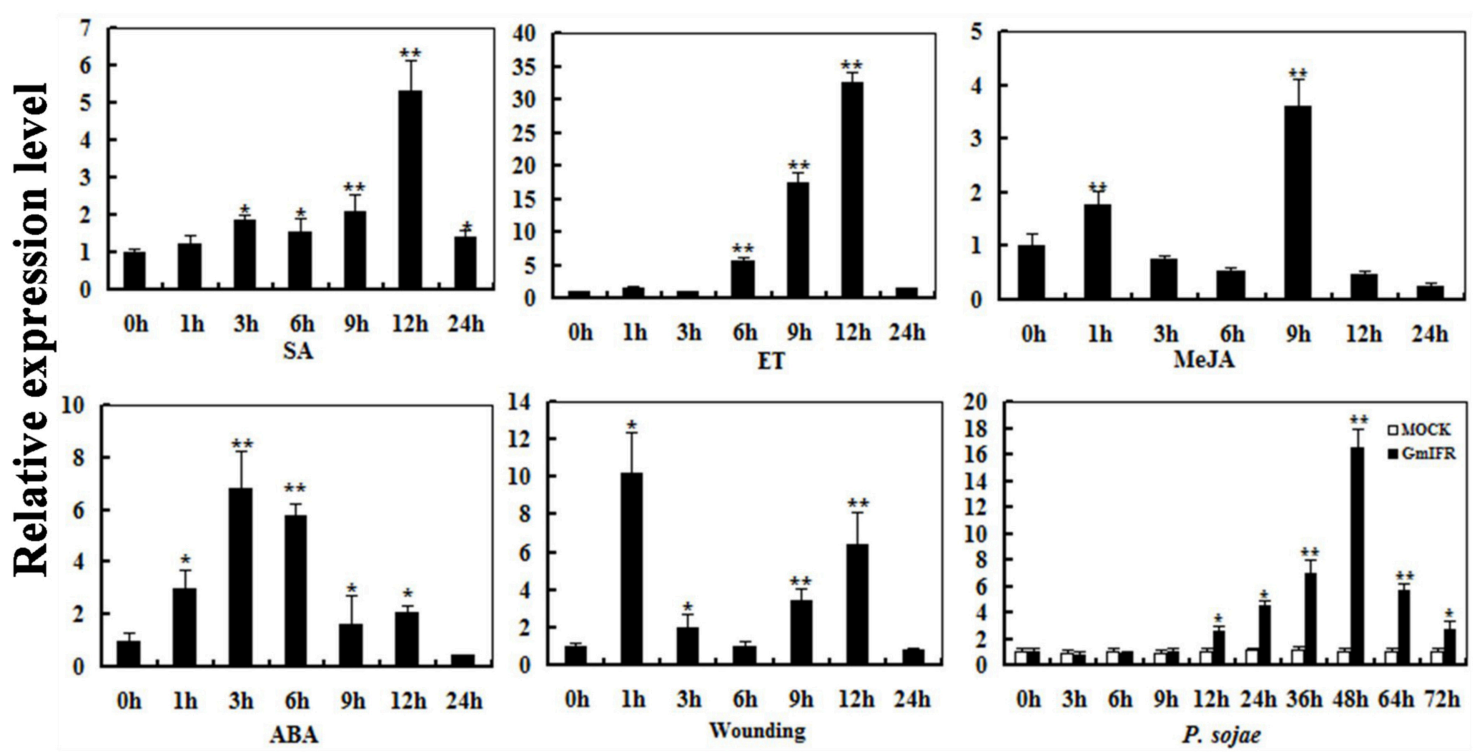

FIGURE 2 | Relative quantities of GmIFR mRNA at various time points post-treatment with ET, SA, MeJA, ABA, wounding, and $P$. sojae. Fourteen-day-old plants were used for treatments and analyses. Water treatment were used as control for $P$. sojae treatments. The amplification of the soybean Actin (GmActin4) gene was used as an internal control to normalize all the data. Relative transcript levels of GmIFR were quantified compared with mock plants at the same time point. The experiment was performed on three biological replicates with their respective three technical replicates and statistically analyzed using Student's $t$-test $\left({ }^{\star} P<0.05 ;{ }^{*} P<0.01\right)$. Bars indicate standard error of the mean.

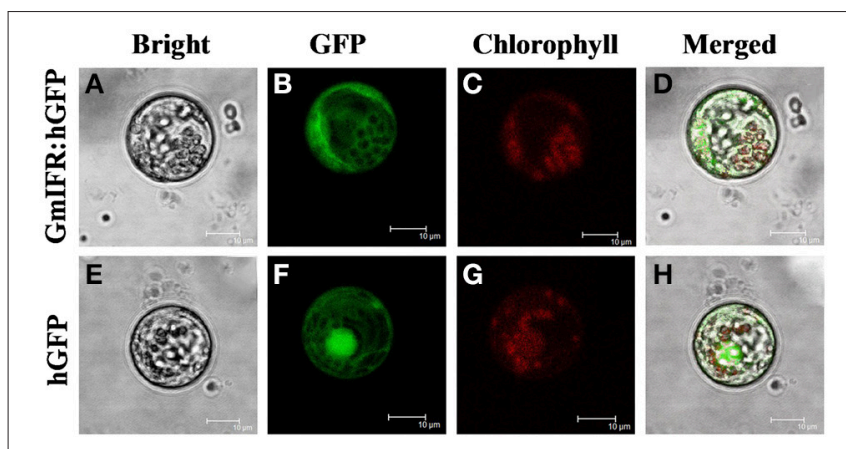

FIGURE 3 | Subcellular localization of the IFR-GFP fusion protein in Soybean protoplasts. GmIFR-GFP expression was driven by the cauliflower mosaic virus $35 \mathrm{~S}$ promoter and transiently expressed in Soybean protoplasts. The images of bright-field $(\mathbf{A}, \mathbf{E})$, the GFP fluorescence (green) only $(\mathbf{B}, \mathbf{F})$, the chlorophyll autofluorescence (red) only (C,G), cytoplasmic marker fluorescence localization, and combined ones $(\mathbf{D}, \mathbf{H})$ are shown. Bars $=10 \mathrm{~mm}$.

\section{The Activity of GmIFR In vitro}

To express GmIFR in Transetta (DE3) cells, the coding sequence of GMIFR was cloned into pET-28a that was an expression vector with a His-tag. Upon induction by IPTG, GmIFR was expressed as a major soluble protein product at $1,2,4,6 \mathrm{~h}$ (Figure 4A, Lane 2, 3, 4, 5). The molecular weight of the purified protein was about $34 \mathrm{kDa}$ in SDS-PAGE, consistent with the calculated molecular mass $(34 \mathrm{kDa})$ (Figure 4A, lane 6). Western blotting of the purified recombinant GmIFR protein confirmed its specific immune reactivity to anti-His antibodies (Figure 4A, lane Western blot).
In order to determine whether GmIFR has isoflavone reductase activity, the enzyme assays of GmIFR was analyzed. The reductase reactions in the presence of NADPH were measured by reversed-phase HPLC: the formation of the product from the substrate. As shown in Figures 4B,C, the protein purified from Transetta (DE3) expressing the fusion protein showed clear isoflavone reductase activity, with $2^{\prime}$ hydroxyformononetin as the substrate. These results proved that GmIFR is an isoflavone reductase.

\section{Overexpression of GmIFR Enhanced Resistance to $P$. sojae in Soybean}

To investigate whether overexpression of GMIFR in soybean has an effect on Phytophthora root rot resistance, the living cotyledons of three T8 transgenic soybean plants (T8-80, T8-88, T8-96) were selected by Real-time PCR (Figure 5A).

As shown in Figure 5B, the cotyledons of the non-transgenic soybean plants detached and exhibited clear and large watersoaked lesions compared with those of the transgenic plants after $72 \mathrm{~h}$ of incubation with $P$. sojae (Figure 5B). The lesion area of the three transgenic lines was significantly $(P<0.01)$ smaller than that of non-transgenic soybean plants at $72 \mathrm{~h}$ after inoculation (Figure 5C). These results indicated that constitutive expression of the GmIFR enhances resistance toward P. sojae.

\section{Expression of GmIFR in Soybean Seed Affects ROS Levels}

It is known that pathogen infection is associated with the production of ROS (Hückelhoven and Kogel, 2003; Soosaar et al., 

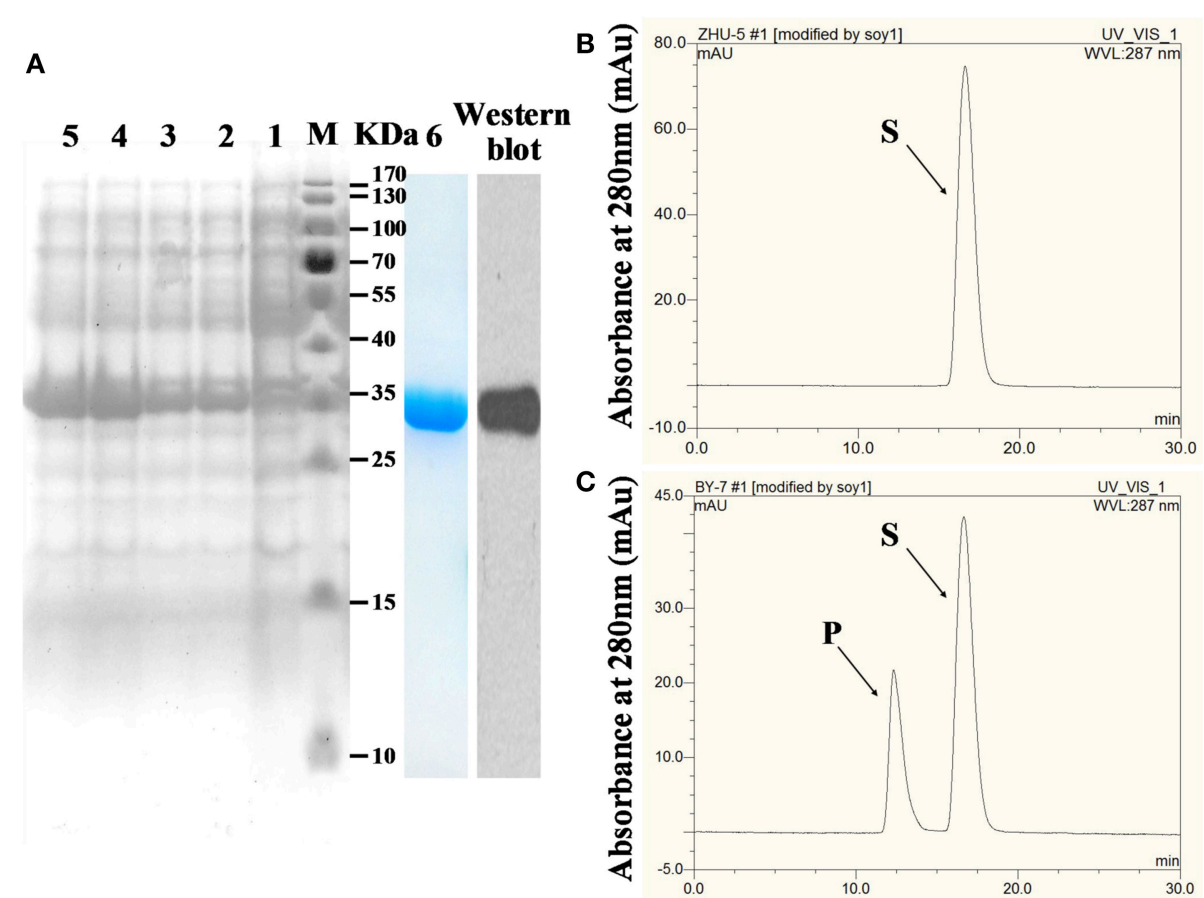

FIGURE 4 | Analysis of GmIFR activity by HPLC. (A) After IPTG induction, Transetta cells containing pET28a-IFR were grown at $37^{\circ} \mathrm{Cfor} 1,2,4,6 \mathrm{~h}$. Lane 1 protein of total cells without IPTG induction, lane 2 protein of total cells with IPTG induction for $1 \mathrm{~h}$, lane 3 protein of total cells with IPTG induction for $2 \mathrm{~h}$, lane 4 induction for $4 \mathrm{~h}$, lane 5 induction for $6 \mathrm{~h}$, lane 6 purified recombinant GmIFR protein with Nickel-CL agarose affinity chromatography and used for enzyme activity assay; $M$, molecular marker; Lane Western blot western blotting of the purified recombinant GmIFR protein with an anti-His tag primary antibody probe. (B) The reaction with 2'-hydroxyformononetin. (C) The reaction harboring the GmIFR protein and with 2'-hydroxyformononetin as the substrate. S, substrate; P, product.

2005; Takabatake et al., 2007; Shetty et al., 2008). Therefore, the ROS relative expression levels was detected in three T8 transgenic soybean plants (T8-31, T8-39, and T8-47) and non-transgenic plants at $0,3,6,12,24,48 \mathrm{~h}$ after incubation with $P$. sojae. The results showed that the relative expression levels of ROS gradually increased in transgenic plants and non-transgenic plants with the incubation period (Figure 6). The relative expression levels of ROS in the transgenic plants were significantly lower than those of non-transgenic plants at the same time point (Figure 6), suggesting that an important role of GmIFR might function as an antioxidant to reduce ROS in soybean.

\section{Overexpression of GmIFR in Soybean Seed Affects Isoflavone and Glyceollins Expression Levels}

It is well known that daidzein, genistein, and glycitein were the essential components of isoflavones (Wang et al., 2014) and IFR was a crucial enzyme involved in the synthesis of the glyceollins from daidzein (Graham et al., 1990; Oliver et al., 2003). Thus, changes in the IFR expression level may cause change of isoflavonoid and glyceollins content in soybean. To study the relationship between isoflavonoid and glyceollins content in soybean seeds and the GMIFR gene expression level, the isoflavonoid content and the relative content of the glyceollins were measured in the seeds of transgenic soybean plants and non-transgenic soybean plants. As shown in Figure 7A, the daidzein levels in the seeds (numbered S-T8-80, S-T8-88, and S-T8-96) developed from the three independent transformed lines T8-80, T8-88, and T8-96 reduced greatly, while levels of genistein and glycitein had little change compared to those of control (Figures 7B,C). The relative content of glyceollins in the transgenic plants was significantly higher than that of nontransgenic plants (Figure 7D), suggesting that an important role of GmIFR involved in the synthesis of the glyceollins from daidzein in soybean.

\section{Over-expressing of GmIFR Affected the Transcriptional Level of Multiple Genes Involved in Phenylpropanal Pathway}

To test whether GmIFR protein could regulate stress-related genes expression in the isoflavonoids synthesis pathway, the expression of GmIFR and three stress-related genes ( $G m P A L, G m 4 C L, G m C H S$ ) was analyzed in transgenic soybean plants and non-transgenic plants using qRT-PCR at $0,6,12,24$, $48,64 \mathrm{~h}$ after incubation with $P$. sojae. As shown in Figure 8, the transcript levels of GmPAL,Gm4CL, GmCHS were induced after incubation with $P$. sojae in transgenic soybean plants and nontransgenic soybean, and the transcript levels of the three genes were significantly higher than those of non-transgenic soybean plants at the same time point. 


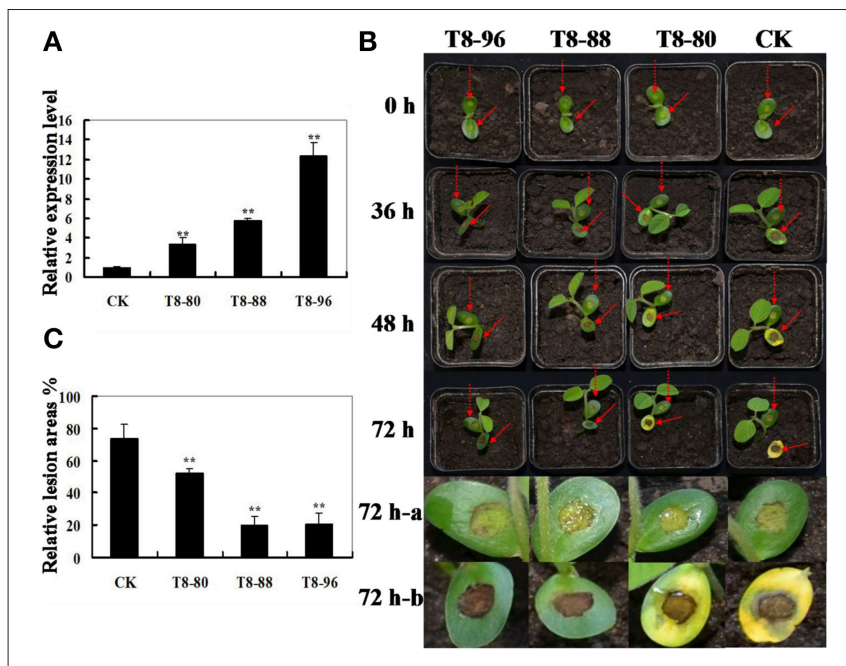

FIGURE 5 | Resistance analysis of GmIFR transgenic soybean plants. (A) qRT-PCR determining the relative abundance of GmIFR (lines T8-80, T8-88, and T8-96) in the transgenic soybean plants. The non-transgenic soybean plants were used as controls. For each sample, three biological replicates were analyzed with their respective three technical replicates and statistically analyzed using Student's $t$-test $\left({ }^{\star *} P<0.01\right)$. Bars indicate standard error of the mean. (B) Disease symptoms after infection with $P$. sojae. Lesions on living cotyledon at $72 \mathrm{~h}$ with $P$. sojae isolate race 1 . For infection assays, three biological replicates were analyzed with their respective technical replicates. And do the same with control. (C) Relative lesion area of transgenic soybean cotyledon infection with $P$. sojae after $72 \mathrm{~h}$. Seventy-two hours a represents the cotyledon of transgenic soybean and non-transgenic soybean infected with V8 juice agar, and $72 \mathrm{~h}-\mathrm{b}$ represents the cotyledon were treated with a $P$. sojae inoculum. The average lesion area of each independent transgenic line $(n=3)$ was calculated and their relative lesion areas are shown in columns after comparison with the average lesion area on non-transgenic soybean. The statistically analyzed using Student's $t$-test $\left({ }^{*} P<0.01\right)$. Bars indicate standard error of the mean.

\section{DISCUSSION}

In this study, we identified a novel GmIFR gene that encodes for a $\mathrm{NAD}(\mathrm{P}) \mathrm{H}$-dependant oxidoreductase, enhances resistance to $P$. sojae when over-expressed in soybean. The gene encoding isoflavonoid reductase (IFR), one of the key enzymes in isoflavonoid phytoalexin biosynthesis, was first cloned from alfalfa (Medicago sativa L.; Paiva et al., 1991). However, there is little knowledge about the biological function of IFR in soybean. Here, we report for the first time that GmIFR transgenic soybean plants inoculated with $P$. sojae display significantly altered responses to pathogen infection.

Plants encounter a range of environmental stresses in their natural environments and have evolved a wide range of mechanisms to cope with them (Dixon and Paiva, 1995; Zhang et al., 2008). There are multiple stress perception and signaling pathways, some of which are specific, whereas others crosstalk at various steps (Kunkel and Brooks, 2002; Chinnusamy et al., 2004; Fujita et al., 2006; Rípodas et al., 2013). It has been reported that higher levels of IFR determining fungal resistance in response to A. rabiei in chickpea (Daniel et al., 1990). In the present study, we demonstrated that overexpression of the GmIFR gene improved resistance to $P$. sojae in soybean.

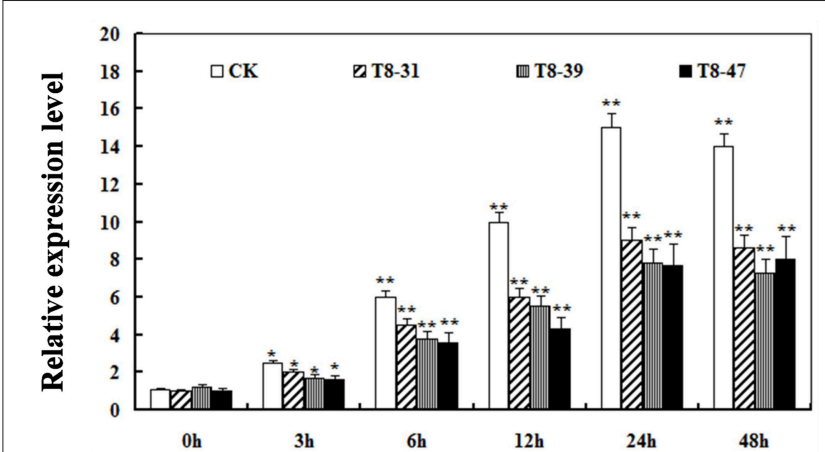

FIGURE 6 | Relative expression levels of reactive oxygen species (ROS) in transgenic soybean plants and non-transgenic soybean plants at 0 , $3,6,12,24,48 \mathrm{~h}$ after $\boldsymbol{P}$. sojae infection. Values are relative to the value of mock plants at the same time point. Statistically significant differences were performed between the overexpression transgenic lines and non-transgenic lines. Three biological replicates with their three technical replicates were averaged and statistically analyzed using Student's $t$-test ${ }^{*} P<0.05$; $\left.{ }^{\star \star} P<0.01\right)$. Bars indicate standard error of the mean.
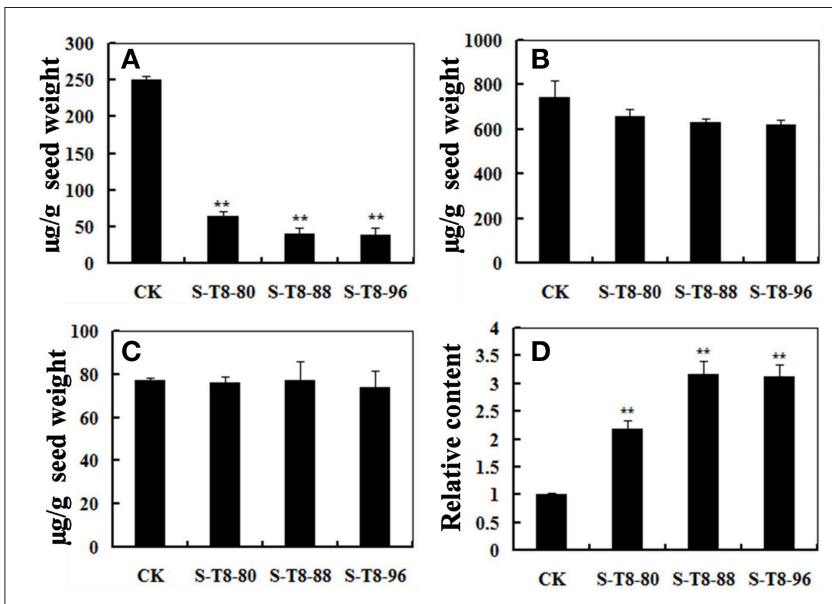

FIGURE 7 | The content of isoflavone components and the relative content of glyceollins in seeds of transgenic and non-transgenic soybeans. (A) The daidzein levels in seeds of transgenic and non-transgenic soybeans. (B) The glycitein levels in seeds of transgenic and non-transgenic soybeans. (C) The genistein levels in seeds of transgenic and non-transgenic soybeans. (D) The relative content of glyceollins in the seeds of transgenic and non-transgenic soybeans. The experiment was performed three biological replicates with their respective three technical replicates and statistically analyzed using Student's $t$-test $\left({ }^{* *} P<0.01\right)$. Bars indicate standard error of the mean.

Moreover, the genes with high sequence similarity to IFRs have been identified in non-leguminous plants and are called isoflavone reductases like (IRL; Shoji et al., 2002). In most cases, IRLs might involve in responses to biotic or abiotic stresses (Shoji et al., 2002; Zhu et al., 2009; Cheng et al., 2013). A recent study suggested that GbIRL is involved in regulating ABA, JA, and ET stress responses in Ginkgo (Cheng et al., 2013). In this study, the expression of GmIFR following various stress treatments was analyzed. The results showed that inoculation with $P$. sojae as biotic stress and woundings as abiotic stress 

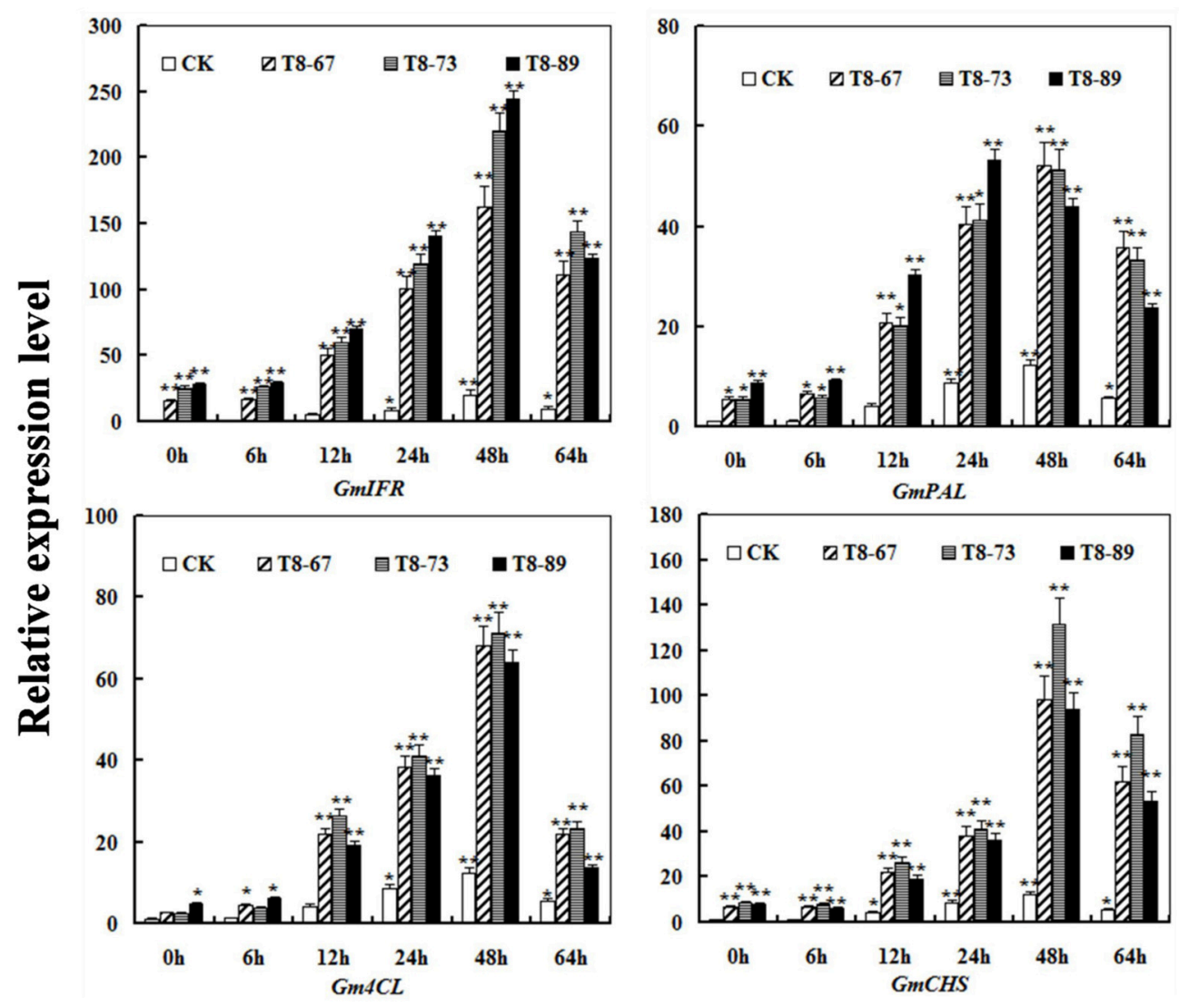

FIGURE 8 | The transcript levels of the three genes (GmPAL, Gm4CL, GmCHS) in GmIFR transgenic and non-transgenic soybean plants after $P$. sojae infection using quantitative RT-PCR analysis. The amplification of the soybean Actin (GmActin4) gene was used as an internal control to normalize all the data. The relative transcript levels of the genes were quantified compared with mock plants at the same time point. The experiment was performed on three biological replicates with their respective three technical replicates and statistically analyzed using Student's $t$-test $\left({ }^{*} P<0.05 ;{ }^{* *} P<0.01\right)$. Bars indicate standard error of the mean.

significantly increased the transcript levels of GmIFR in soybean plants. The expression of GmIFR was also induced by treatments with SA, ET, JA, and ABA. ABA is a phytohormone that is extensively involved in responses to abiotic stresses such as drought and low temperature, as well as osmotic stress (Skriver and Mundy, 1990). In contrast, the phytohormones SA, JA, and ET play central roles in biotic stress signaling following pathogen infection (Pieterse et al., 2009; Robert-Seilaniantz et al., 2011; Sugano et al., 2013). Therefore, it is possible to speculate that GmIFR likely plays an important role in responsive to biotic stresses in soybean. Moreover, it has been reported that IFR proteins as the key enzymes can catalyze reductase reactions (Paiva et al., 1991; Gang et al., 1999). For instance, IFR can convert 2'-hydroxyformononetin of the isoflavonoid substrates to (3R)-vestitone in alfalfa (Paiva et al., 1991). In Ginkgo, the recombinant GbIRL1 protein could catalyze the formation of the TDDC, IDDDC from DDDC, DDC (Cheng et al., 2013). In this work, we determined the recombinant GmIFR protein could catalyze oxidoreductase reaction using enzyme assays. Therefore, it is proven that GmIFR has isoflavone reductase activity.

There have been some reports about the inhibition of glyceollin on several lines of pathogen species (Lyne et al.,
1976; Kim et al., 2010a, 2012; Nwachukwu et al., 2013). More specifically, the glyceollin can resist to Phytophthora megasperma var. sojae in soybean (Lyne et al., 1976; Hahn et al., 1985; Lygin et al., 2010, 2013). In addition, Boue and Raina (2003) noted that the fungi Aspergillus flavus, Aspergillus niger, Aspergillus oryzae, and Aspergillus flavus were all able to induce glyceollin in soybean. Glyceollin revealed a remarkable antimicrobial effect against Phytophthora capsici and Sclerotinia scrotiorum by Kim et al. (2010a). Moreover, It has been reported that IFR was a key enzyme involved in the synthesis of the glyceollins from daidzein which was the essential component of the isoflavones (Graham et al., 1990; Oliver et al., 2003). In this work, we detected the content of daidzein, genistein, glycitein and the relative content of glyceollins in transgenic soybean seeds and nontransgenic soybean seeds. The results showed that the daidzein content greatly reduced in transgenic soybean seeds, while levels of genistein and glycitein had little change compared to those of the non-transgenic soybean seeds. The relative content of glyceollins in the transgenic plants was significantly higher than that of non-transgenic plants. Therefore, we suggested that GmIFR might improve the resistance to $P$. sojae in soybean when overexpression likely by increasing the accumulation of 
glyceollins. It has been reported that the biosynthesis of glyceollin via the isoflavonoid branch of the phenylpropanoid pathway (Ng et al., 2011) and glyceollin as a phytoalexin could response to pathogen invasion (Hahn et al., 1985; Lygin et al., 2010; Kim et al., 2012). PAL, 4CL, and CHS play an important role in the isoflavonoid branch of the phenylpropanoid pathway (Vogt, 2010; Yi et al., 2010). Here, we studied the transcript levels change of the three genes (GmPAL, GmCHS, Gm4CL) after incubation with $P$. sojae in transgenic and non-transgenic soybean plants. We found that these genes were induced after incubation with $P$. sojae and there was up-regulation of the transcript in transgenic soybean plants. We speculated that the three genes ( $G m P A L, G m C H S, G m 4 C L$ ) might play a cooperation role on the biosynthesis of glyceollin which might improve resistance to $P$. sojae in soybean.

Plants experience a variety of environmental stresses likely leading to the generation of ROS (Sies, 1991). Although ROS may be essential to maintain homoeostasis, the generation of ROS, within certain boundaries, is harmful at the high concentration (Kim et al., 2012). However, glyceollins as major isoflavonoid phytoalexin in leguminous plants, showed strong antioxidant activity and ROS scavenging potential when assessed by an in vitro model (Kim et al., 2010a, 2012). Moreover, Kim et al. (2010b) reported that overexpression of OsIRL in transgenic rice plants promotes resistance to ROS. A possible interpretation is that the IRLs contain a putative NAD (P) domain related to oxidation/reduction properties (Babiychuk et al., 1995; Petrucco et al., 1996). The different roles of ROS in plants interactions with special emphasis on fungal and oomycete pathogens have also been reported (Shetty et al., 2008). For example, Blumeria graminis f. sp. hordei can induce ROS accumulation in barley (Hückelhoven and Kogel, 2003) and Septoria tritici can induce ROS accumulation in wheat (Shetty et al., 2003). Thus, it can be deduced that the plants can improve resistance to pathogen by scavenge excess ROS. Consistent with these, we detected the relative expression levels of ROS in transgenic soybean plants and non-transgenic plants after incubation with $P$. sojae, and found that the

\section{REFERENCES}

Babiychuk, E., Kushnir, S., Belles-Boix, E., Van Montagu, M., and Inz'e, D. (1995). Arabidposis thaliana NADPH oxidoreductase homologs confer tolerance of yeast toward the thiol-oxidizing drug diamide. J. Biol. Chem. 270, 26224-26231. doi: $10.1074 /$ jbc.270.44.26224

Banks, S. W., and Dewick, P. M. (1983). Biosynthesis of glyceollins I, II and III in soybean. Phytochemistry 22, 2729-2733. doi: 10.1016/S0031-9422(00) 97682-9

Boue, S. M., Carter, C. H., Ehrlich, K. C., and Clevel, T. E. (2000). Induction of the soybean phytoalexins coumestrol and glyceollin by Aspergillus. J. Agric. Food Chem. 48, 2167-2172. doi: 10.1021/jf9912809

Boue, S. M., and Raina, A. K. (2003). Effects of plant flavonoids on fecundity, survival, and feeding of the Formosan subterranean termite. J. Chem. Ecol. 29, 2575-2584. doi: 10.1023/A:1026318203775

Cheng, H., Li, L. L., Xu, F., Wang, Y., Yuan, H. H., Wu, C. H., et al. (2013). Expression patterns of an isoflavone reductase-like gene and its possible roles in secondary metabolism in Ginkgo biloba. Plant Cell Rep. 32, 637-650. doi: $10.1007 /$ s00299-013-1397-2 relative expression levels of ROS in transgenic plants were significantly lower than those of control plants. Overexpression of GmIFR in transgenic soybean plants can increase the content of glyceollins or GmIFR itself behave as an antioxidant to scavenge ROS, which might improve resistance to $P$. sojae in soybean.

\section{ACKNOWLEDGMENTS}

The research was supported through funding from the Heilongjiang Province outstanding youth fund (JC201308), NSFC Projects (30971811, 31071439, 31171577, 31101167), Natural Science Foundation of Heilongjiang Province (C2015010), the Specialized Research Fund for the Doctoral Program of Higher Education (20112325120005), the Science and Technology Innovation Project in Harbin (2012RFQXN011, 2012RFXXN019), and the Research Fund for Young Teachers through NEAU (2012 RCB 08).

\section{SUPPLEMENTARY MATERIAL}

The Supplementary Material for this article can be found online at: http://journal.frontiersin.org/article/10.3389/fpls.2015. 01024

\section{Supplementary Table S1 | Oligonucleotide primers used in this study.}

Supplementary Figure S1 | The nucleotide sequence of GmIFR cDNA together with its predicted amino acid sequence. The NAD $(P)$ domain is underlined.

Supplementary Figure S2 | Phylogenetic analysis and sequence alignment of GmIFR in the leguminous plants genome. (A) The predicted

three-dimensional model of the IFR. (B) Phylogenetic relationships of GmIFR with IFRs proteins; the phylogenetic tree was constructed using amino acid sequences of the IFRs protein from other species. The plant species and GenBank accession numbers were as follows: Glycine max GmIFR (NM_001254100); Phaseolus vulgaris PvIFR (XP_007156276.1), Cicer arietinum CalFR (XP_004509553.1), Medicago truncatula MtIRF (AFK37791.1), Lotus japonicus LjIFR (BAF34845.1), Glycine soja GsIFR (KHN36092.1), Medicago sativa MsIFR (CAA41106), Pisum sativum PsIFR (P52576.1). (C) The conserved NAD (P) domain of the IFR group proteins.
Chinnusamy, V., Schumaker, K., and Zhu, J. K. (2004). Molecular genetic perspectives on cross-talk and specificity in abiotic stress signalling in plants. J. Exp. Bot. 55, 225-236. doi: 10.1093/jxb/erh005

Cooper, J. D., Qiu, F., and Paiva, N. L. (2002). Biotransformation of an exogenously supplied isoflavonoid by transgenic tobacco cells expressing alfalfa isoflavone reductase. Plant Cell Rep. 20, 876-884. doi: 10.1007/s00299-0010404-1

Daniel, S., Tiemann, K., Wittkampf, U., Bless, W., Hinderer, W., and Barz, W. (1990). Elicitor-induced metabolic changes in cell cultures of chickpea (Cicer arietinum L.) cultivars resistant and susceptible to Ascochyta rabiei. Planta 182, 270-278.

Dixon, R. A., and Paiva, N. L. (1995). Stress-induced phenylpropanoid metabolism. Plant Cell 7, 1085-1097. doi: 10.1105/tpc.7.7.1085

Dou, D. L., Wang, B. S., Zhu, S. W., Tang, Y. X., Wang, Z. X., Sun, J. S., et al. (2003). Transgenic tobacco with NDR1 gene improved its resistance to two fungal disease. Sci. Agric. Sin. 36, 1120-1124.

Fehr, W. R., Caviness, C. E., Burmood, D. T., and Pennington, J. (1971). Stage of development descriptions for soybeans, Glycine $\max$ (L.) Merrill. Crop Sci. 11, 929-931. doi: 10.2135/cropsci1971.0011183X001100060051x 
Fischer, D., Ebeanau-Jehle, C., and Grisebach, H. (1990). Phytoalexin synthesis in soybean: purification and characterization of NADPH: 20-hydroxydaidzein oxidoreductase from elicitor-challenged soybean cellcultures. Arch. Biochem. Biophys. 276, 390-395. doi: 10.1016/0003-9861(90)90737-J

Fujita, M., Fujita, Y., Noutoshi, Y., Takahashi, F., Narusaka, Y., YamaguchiShinozaki, K., et al. (2006). Crosstalk between abiotic and biotic stress responses: a current view from the points of convergence in the stress signaling networks. Curr. Opin. Plant Biol. 9, 436-442. doi: 10.1016/j.pbi.2006.05.014

Gang, D. R., Kasahara, H., Xia, Z. Q., Vander Mijnsbrugge, K., Bauw, G., Boerjan, W., et al. (1999). Evolution of plant defense mechanisms: relationships of phenylcoumaran benzylic ether reductases to pinoresinol lariciresinol and isoflavone reductases. J. Biol. Chem. 274, 7516-7527. doi: $10.1074 /$ jbc.274.11.7516

Graham, T. L., Kim, J. E., and Graham, M. Y. (1990). Role of constitutive isoflavone conjugates in the accumulation of glyceollin in soybean infected with Phytophthora megasperma. Mol. Plant Microbe 3, 157-166. doi: 10.1094/MPMI3-157

Guo, L., Dixon, R. A., and Paiva, N. L. (1994). Conversion of vestitone to medicarpin in alfalfa (Medicago sativa L.) is catalyzed by two independent enzymes. J. Biol. Chem. 269, 22372-22378.

Hahn, M. G., Bonhoff, A., and Grisebach, H. (1985). Quantitative localization of the phytoalexin glyceollinI in relation to fungal hyphae in soybean roots infected with Phytophthora megasperma f. sp. glycinea. Plant Physiol. 77, 591-601. doi: 10.1104/pp.77.3.591

Hückelhoven, R., and Kogel, K. H. (2003). Reactive oxygen intermediates in plant microbe interactions: who is who in powdery mildew resistance? Planta 216, 891-902. doi: 10.1007/s00425-003-0973-z

Kim, H. J., Lim, J. S., Kim, W. K., and Kim, J. S. (2012). Soybean glyceollins: biological effects and relevance to human health. Proc. Nutr. Soc. 71, 166-174. doi: $10.1017 /$ S0029665111003272

Kim, H. J., Suh, H. J., Kim, J. H., Park, S., Joo, Y. C., and Kim, J. S. (2010a). Antioxidant activity of glyceollins derived from soybean elicited with Aspergillus sojae. Agric. Food Chem. 58, 11633-11638. doi: 10.1021/jf102829z

Kim, S. G., Kim, S. T., Wang, Y. M., Kim, S. K., Lee, C. H., Kim, K. K., et al. (2010b). Overexpression of rice isoflavone reductase-like gene (OsIRL) confers tolerance to reactive oxygen species. Physiol. Plant 138, 1-9. doi: 10.1111/j.1399-3054.2009.01290.x

Kim, S. T., Cho, K. S., Kim, S. G., Kang, S. Y., and Kang, K. Y. (2003). A rice isoflavone reductase-like gene, $O s I R L$, is induced by rice blast fungal elicitor. Mol. Cells 16, 224-231.

Kunkel, B. N., and Brooks, D. M. (2002). Cross talk between signaling pathways in pathogen defense. Curr. Opin. Plant Biol. 5, 325-331. doi: 10.1016/S13695266(02)00275-3

Lers, A., Bud, S., Lomanic, E., Droby, S., and Chalutz, E. (1998). The expression of a grapefruit gene encoding an isoflavone reductase-like protein is induced in response to UV-irradiation. Plant Mol. Biol. 36, 847-856. doi: 10.1023/A:1005996515602

Lin, W. (1983). Isolation of mesophyll protoplasts from mature leaves of soybeans. Plant Physiol. 73, 1067-1069. doi: 10.1104/pp.73.4.1067

Lygin, A. V., Hill, C. B., Zernova, O. V., Crull, L., Widholm, J. M., Hartman, G. L., et al. (2010). Response of soybean pathogens to glyceollin. Phytopathology 100, 897-903. doi: 10.1094/PHYTO-100-9-0897

Lygin, A. V., Zernova, O. V., Hill, C. B., Kholina, N. A., Widholm, J. M., Hartman, G. L., et al. (2013). Glyceollin is an important component of soybean plant defense against Phytophthora sojae and Macrophomina phaseolina. Phytopathology 103, 984-994. doi: 10.1094/PHYTO-12-12-0328-R

Lyne, R. L., Mulheirn, L. J., and Leworthy, D. P. (1976). New pterocarpinoid phytoalexins of soybean. J. Chem. Soc. Chem. Commun. 13, 497-498. doi: $10.1039 / \mathrm{c} 39760000497$

Morris, P. F., Savard, M. E., and Ward, E. W. B. (1991). Identification and accumulation of isoflavonoids and isoflavone glucosides in soybean leaves and hypocotyls in resistance responses to Phytophthora megasperma f. sp. glycines. Physiol. Mol. Plant 39, 229-224. doi: 10.1016/0885-5765(91)9 0006-4

Morrison, R. H., and Thorne, J. C. (1978). Inoculation of detached cotyledons for screening soybeans against two races of Phytophthora megasperma var. sojae. Crop Sci. 18, 1089-1091. doi: 10.2135/cropsci1978.0011183X00180006 0049x
Ng, T. B., Ye, X. J., Wong, J. H., Fang, E. F., Chan, Y. S., Pan, W. L., et al. (2011). Glyceollin, a soybean phytoalexin with medicinal properties. Appl. Microbiol. Biotechnol. 90, 59-68. doi: 10.1007/s00253-011-3169-7

Nwachukwu, I. D., Luciano, F. B., and Udenigwe, C. C. (2013). The inducible soybean glyceollin phytoalexins with multifunctional health-promoting properties. Food Res. Int. 54, 1208-1216. doi: 10.1016/j.foodres.2013. 01.024

Oliver, Y., Shi, J., Hession, A. O., Maxwell, C. A., McGongigle, B., and Odell, J. T. (2003). Metabolic engineering to increase isoflavone biosynthesis in soybean seed. Phytochemistry 63, 753-763. doi: 10.1016/S0031-9422(03)00345-5

Paiva, N. L., Edwards, R., Sun, Y. J., Hrazdina, G., and Dixon, R. A. (1991). Stress responses in alfalfa (Medicago sativa L.) 11. Molecular cloning and expression of alfalfa isoflavone reductase, a key enzyme of isoflavonoid phytoalexin biosynthesis. Plant Mol. Biol. 17, 653-667. doi: 10.1007/BF00037051

Partridge, J. E., and Keen, N. T. (1977). Soybean phytoalexins: rates of synthesis are not regulated by activation of initial enzymes in flavonoid biosynthesis. J. Physiol. Biochem. 67, 50-55. doi: 10.1094/phyto-67-50

Paz, M. M., Shou, H. X., Guo, Z. B., Zhang, Z. Y., Banerjee, A. K., and Wang, K. (2004). Assessment of conditions affecting Agrobacterium-mediated soybean transformation using the cotyledonary node explant. Euphytica 136, 167-179. doi: 10.1023/B:EUPH.0000030669.75809.dc

Petrucco, S., Bolchi, A., Foroni, C., Percudani, R., Rossi, G. L., and Ottonello, S. (1996). A maize gene encoding an NADPH binding enzyme highly homologous to isoflavone reductases is activated in response to sulfur starvation. Plant Cell 8, 69-80. doi: $10.1105 /$ tpc.8.1.69

Pieterse, C. M. J., Leon-Reye, A., Van der Ent, S., and Van Wees S. C. (2009). Networking by smallmolecule hormones in plant immunity. Nat. Chem. Biol. 5, 308-316. doi: 10.1038/nchembio. 164

Qian, H. F., Chen, W., Sun, L. W., Jin, Y. X., Liu, W. P., and Fu, Z. W. (2009). Inhibitory effects of paraquat on photosynthesis and the response to oxidative stress in Chlorella vulgaris. Ecotoxicology 18, 537-543. doi: 10.1007/s10646-0090311-8

Rípodas, C., Via, V. D., Aguilar, O. D., Zanetti, M. E., and Blanco, F. A. (2013). Knock-down of a member of the isoflavone reductase gene family impairs plant growth and nodulation in Phaseolus vulgaris. Plant Physiol. Bioch. 68, 81-89. doi: 10.1016/j.plaphy.2013.04.003

Robert-Seilaniantz, A., Grant, M., and Jones, J. D. G. (2011). Hormone crosstalk in plant disease and defense: more than just jasmonate-salicy late antagonism. Annu. Rev. Phytopathol. 49, 317-343. doi: 10.1146/annurev-phyto-073009114447

Shetty, N. P., Kristensen, B. K., Newman, M. A., Møller, K., Gregersen, P. L., and Jørgensen, H. J. L. (2003). Association of hydrogen peroxide with restriction of Septoria tritici in resistant wheat. Physiol. Mol. Plant 62, 333-346. doi: 10.1016/S0885-5765(03)00079-1

Shetty, N. P., Lyngs Jørgensen, H. J., Jensen, J. D., Collinge, D. B., and Shetty, H. S. (2008). Roles of reactive oxygen species in interactions between plants and pathogens. Eur. J. Plant Pathol. 121, 267-280. doi: 10.1007/s10658-008-9302-5

Shoji, T., Winz, R., Iwase, T., Nakajima, K., Yamada, Y., and Hashimoto, T. (2002). Expression patterns of two tobacco isoflavone reductase-like genes and their possible roles in secondary metabolism in tobacco. Plant Mol. Biol. 50, 427-440. doi: 10.1023/A:1019867732278

Sies, H. (1991). Oxidative stress: from basic research to clinical application. Am. J. Med. 91, 31-38. doi: 10.1016/0002-9343(91)90281-2

Skriver, K., and Mundy, J. (1990). Gene expression in response to abscisic acid and osmotic stress. Plant Cell 2:503. doi: 10.1105/tpc.2.6.503

Somerville, C., and Somerville, S. (1999). Plant functional genomics. Science 285 , 380-383. doi: 10.1126/science.285.5426.380

Soosaar, J. L. M., Burch-Smith, T. M., and Dinesh-Kumar, S. P. (2005). Mechanisms of plant resistance to viruses. Nat. Rev. Microbiol. 3, 789-798. doi: $10.1038 /$ nrmicrol239

Sugano, S., Sugimoto, T., Takatsuji, H., and Jiang, J. (2013). Induction of resistance to Phytophthora sojae in soybean (Glycine max) by salicylic acid and ethylene. Plant Pathol. 62, 1048-1056. doi: 10.1111/ppa.12011

Sun, Y. J., Wu, Q. D., VanEtten, H. D., and Hrazdina, G. (1991). Stereoisomerism in plant disease resistance: induction and isolation of the 7, 20-dihydroxy-40, 50methylenedioxyisoflavone reductase, an enzyme introducing chirality during synthesis of isoflavanoid phytoalexins in pea (Pisum sativum L). Arch. Biochem. Biophys. 284, 167-173. doi: 10.1016/0003-9861(91)90279-R 
Takabatake, R., Ando, Y., Seo, S., Katou, S., Tsuda, S., Ohashi, Y., et al. (2007). MAP kinases function downstream of HSP 90 and upstream of mitochondria in TMV resistance gene $N$-mediated hypersensitive cell death. Plant Cell Physiol. 48, 498-510. doi: 10.1093/pcp/pcm021

Tamura, K., Dudley, J., Nei, M., and Kumar, S. (2007). MEGA4: molecular evolutionary genetics analysis (MEGA) software version 4.0. Mol. Biol. Evol. 24, 1596-1599. doi: 10.1093/molbev/msm092

Vogt, T. (2010). Phenylpropanoid biosynthesis. Mol. Plant 3, 2-20. doi: $10.1093 / \mathrm{mp} / \mathrm{ssp} 106$

Wang, X. Q., He, X. Z., Lin, J. Q., Shao, H., Chang, Z. Z., and Dixon, R. A. (2006). Crystal structure of isoflavone reductase from alfalfa (Medicago sativa L.). J. Mol. Biol. 358, 1341-1352. doi: 10.1016/j.jmb.2006.03.022

Wang, Y., Han, Y. P., Teng, W. L., Zhao, X., Li, Y. G., Wu, L., et al. (2014). Expression quantitative trait loci infer the regulation of isoflavone accumulation in soybean (Glycine max L. Merr.) seed. BMC Genomics 15:680. doi: 10.1186/1471-2164-15-680

Ward, E. W. B., Lazarovits, G., Unwin, C. H., and Buzzell, R. I. (1979). Hypocotyl reactions and glyceollin in soybeans inoculated with zoospores of Phytophthora megaspuma var. sojae. Phytopathology 69, 951-955. doi: 10.1094/Phyto69-951

Xu, P. F., Chen, W. Y., Lv, H. Y., Fan, S. J., Wang, X., Jiang, L. Y., et al. (2012). Differentially expressed genes of soybean during infection by Phytophthora sojae. J. Integr. Agric. 11, 368-377. doi: 10.1016/S2095-3119(12) 60021-5

Yi, J. X., Derynck, M. R., Li, X. Y., Telmer, P., Marsolais, F., and Dhaubhadel, S. (2010). A single-repeat MYB transcription factor, GmMYB176, regulates CHS8 gene expression and affects isoflavonoid biosynthesis in soybean. Plant J. 62, 1019-1034. doi: 10.1111/j.1365-313x.2010.04214.x

Yoo, S. D., Cho, Y. H., and Sheen, J. (2007). Arabidopsis mesophyll protoplasts: a versatile cell system for transient gene expression analysis. Nat. Protoc. 2, 1565-1572. doi: 10.1038/nprot.2007.199
Yoshikawa, M., Yamauchi, K., and Masago, H. (1978). Glyceollin: its role in restricting fungal growth in resistant soybean hypocotyls infected with Phytophthora megasperma var. sojae. Physiol. Mol. Plant 12, 73-82. doi: 10.1016/0048-4059(78)90020-6

Zeng, G. L., Li, D. M., Han, Y. P., Teng, W. L., Wang, J., Qiu, L. Q., et al. (2009). Identification of QTL underlying isoflavone contents in soybean seeds among multiple environments. Theo. Appl. Genet. 118, 1455-1463. doi: 10.1007/s00122-009-0994-5

Zhang, G. Y., Chen, M., Chen, X. P., Xu, Z. S., Guan, S., Li, L. C., et al. (2008). Phylogeny, gene structures, and expression patterns of the ERF gene family in soybean (Glycine max L.). J. Exp. Bot. 59, 4095-4107. doi: 10.1093/jxb/ern248

Zhang, S. Z., Xu, P. F., Wu, J. J., Allen, X., Zhang, J. X., Li, W. B., et al. (2010). Races of Phytophthora sojae and their virulences on commonly grown soybean varieties in Heilongjiang, China. Plant Dis. 94, 87-91. doi: 10.1094/PDIS-94-10087

Zhu, Q., Guo, T., Sui, S., Liu, G., Lei, X., Luo, L., et al. (2009). Molecular cloning and characterization of a novel isoflavone reductase-like gene $(F c I R L)$ from high flavonoids-producing callus of Fagopyrum cymosum. Acta. Pharmacol. Sin. 44, 809-819.

Conflict of Interest Statement: The authors declare that the research was conducted in the absence of any commercial or financial relationships that could be construed as a potential conflict of interest.

Copyright (๑) 2015 Cheng, Li, Dong, Zhang, Fan, Jiang, Wang, Xu and Zhang. This is an open-access article distributed under the terms of the Creative Commons Attribution License (CC BY). The use, distribution or reproduction in other forums is permitted, provided the original author(s) or licensor are credited and that the original publication in this journal is cited, in accordance with accepted academic practice. No use, distribution or reproduction is permitted which does not comply with these terms. 